\title{
WRF-CMAQ two-way coupled system with aerosol feedback: software development and preliminary results
}

\author{
D. C. Wong ${ }^{1}$, J. Pleim ${ }^{1}$, R. Mathur ${ }^{1}$, F. Binkowski ${ }^{2}$, T. Otte ${ }^{1}$, R. Gilliam ${ }^{1}$, G. Pouliot ${ }^{1}$, A. Xiu ${ }^{2}$, J. O. Young ${ }^{1}$, and \\ D. Kang ${ }^{3}$ \\ ${ }^{1}$ US Environmental Protection Agency, Research Triangle Park, NC, USA \\ ${ }^{2}$ University of North Carolina, Chapel Hill, NC, USA \\ ${ }^{3}$ Computer Sciences Corporation, Research Triangle Park, NC, USA
}

Correspondence to: D. C. Wong (wong.david-c@epa.gov)

Received: 27 July 2011 - Published in Geosci. Model Dev. Discuss.: 22 September 2011

Revised: 19 February 2012 - Accepted: 27 February 2012 - Published: 12 March 2012

\begin{abstract}
Air quality models such as the EPA Community Multiscale Air Quality (CMAQ) require meteorological data as part of the input to drive the chemistry and transport simulation. The Meteorology-Chemistry Interface Processor (MCIP) is used to convert meteorological data into CMAQ-ready input. Key shortcoming of such one-way coupling include: excessive temporal interpolation of coarsely saved meteorological input and lack of feedback of atmospheric pollutant loading on simulated dynamics. We have developed a two-way coupled system to address these issues. A single source code principle was used to construct this two-way coupling system so that CMAQ can be consistently executed as a stand-alone model or part of the coupled system without any code changes; this approach eliminates maintenance of separate code versions for the coupled and uncoupled systems. The design also provides the flexibility to permit users: (1) to adjust the call frequency of WRF and CMAQ to balance the accuracy of the simulation versus computational intensity of the system, and (2) to execute the two-way coupling system with feedbacks to study the effect of gases and aerosols on short wave radiation and subsequent simulated dynamics. Details on the development and implementation of this two-way coupled system are provided. When the coupled system is executed without radiative feedback, computational time is virtually identical when using the Community Atmospheric Model (CAM) radiation option and a slightly increased $(\sim 8.5 \%)$ when using the Rapid Radiative Transfer Model for GCMs (RRTMG) radiation option in the coupled system compared to the offline WRF-CMAQ system. Once the feedback mechanism is turned on, the ex-
\end{abstract}

ecution time increases only slightly with CAM but increases about $60 \%$ with RRTMG due to the use of a more detailed Mie calculation in this implementation of feedback mechanism. This two-way model with radiative feedback shows noticeably reduced bias in simulated surface shortwave radiation and 2-m temperatures as well improved correlation of simulated ambient ozone and $\mathrm{PM}_{2.5}$ relative to observed values for a test case with significant tropospheric aerosol loading from California wildfires.

\section{Introduction}

3-D chemical transport models (CTMs) that are used in air quality research and regulatory applications are driven by $3-$ D meteorological fields provided by a priori runs of a meteorology model. Historically, the CTMs and meteorological models were developed over several decades along independent tracks with little regard for computational, numerical, or even scientific consistency between the two modeling systems. In recent years, however, there have been several efforts to combine meteorological and chemical transport models into single interactive systems (Grell and Baklanov, 2011). A primary driver for this trend has been the need to include the direct and indirect feedback effects of gases and aerosols on radiative forcing. While these feedback effects are mainly important for climate applications, it is becoming evident that they have substantial effects on local meteorology and air quality in polluted regions (Jacobson et al., 1996; Mathur et al., 1998; Xiu et al., 1999). Zhang (2008) 
has provided an overview of several coupled meteorologychemistry models including the WRF/chem (Grell et al., 2005) model in which chemistry has been added into the Weather Research and Forecasting model (Skamarock et al., 2008) at the science process level. Another approach is to couple historically independent meteorology and chemical transport models into a single executable. Advantages of this approach include maintaining consistency with existing separate loose coupled meteorology-chemistry systems that are being continuously and extensively applied and evaluated. Furthermore, the numerical and computational techniques employed in meteorology models and CTMs differ considerably because of the greater need for strict mass conservation and positive-definiteness of transported scalars in the CTM. Also, CTMs generally use fractional integration of various processes while meteorology models use time split integration of all process rates.

The development of the Community Multiscale Air Quality (CMAQ) (Byun and Schere, 2006) modeling system was initiated in the 1990s by the US Environmental Protection Agency (EPA); and the system has continued to evolve (Foley et al., 2010). The model system has benefited from a diverse user community with over 2000 users from 90 different countries. CMAQ has been and continues to be extensively used to provide guidance in rulemaking such as CAIR (Clean Air Interstate Rule,http://www.epa.gov/cair/), by state and local agencies for air quality management analyses such as SIP (State Implementation Plan), by academia and industry for studying relevant atmospheric processes and model applications. CMAQ has also been adapted into the real-time US National Air Quality Forecasting system (AQF) (Otte et al., 2005) and has been running operationally at National Weather Service since 2003 and was recently deployed for forecasting air quality for the 2010 Shanghai World Expo (Wang et al., 2010).

In general, meteorological models are not built for air quality simulation purposes. Hence, the meteorological model might not have the same map projection, coordinate system and grid format, and layer structure as the air quality model. The CMAQ model uses the Meteorology-Chemistry Interface Processor (MCIP) (Otte and Pleim, 2010) to bridge this gap by providing transformed CMAQ-ready meteorological data. The transformation includes unit conversion, format conversion, vertical grid resolution related interpolation, as well as calculations to create additional diagnostic variables that are required in CMAQ but not available in the meteorology model output. Typically, MCIP produces hourly meteorological data, based on storage requirement considerations, as input to CMAQ.

The flow of information in this one-way coupled system is (Fig. 1a): run a meteorological model, like the FifthGeneration Pennsylvania State University-National Center for Atmospheric Research Mesoscale Model (MM5) (Grell et al., 1994) or the Weather Research and Forecasting (WRF) model (Skamarock, 2008; Michalakes et al., 2005), process the meteorological model's output using MCIP, then run the CMAQ air quality model using the MCIP output. This whole coupling approach has been widely used in the research community as well as in the real-time National Air Quality Forecasting system (Otte et al., 2005), but it has several potential shortcomings. First, the integration time step of CMAQ is much finer than the typical hourly available meteorological data. Interpolation is used to handle this issue; however, interpolation accuracy is a problem for meteorological variables, such as wind direction and speed that are key variables for pollutant transport and dispersion. Additionally, at fine horizontal resolutions $(<10 \mathrm{~km})$, the need for more frequent meteorological information (relative to the typical hourly resolution) becomes critical for consistently representing transport (advective, turbulent, and cloud) processes. Second, the lack of aerosol feedback from CMAQ to the meteorological model is an important omission. For instance, CMAQ is able to compute the concentration, composition, and size distribution of particulate matter (aerosol) in the atmosphere. The presence of aerosols in the atmosphere affects the radiation which in turn affects the photolysis rates which dictate atmospheric photo-chemistry, surface temperature that can affect thermally driven atmospheric chemical reactions, planetary boundary layer (PBL) height which dictates dilution and dispersion of pollutants, and even cloud formation. The response of the meteorological model to aerosol loading can be significant under conditions of significant pollution loading. Without such feedback, errors may be introduced into the meteorological model. Third, netCDF file format (32-bits precision) is used to store the intermediate data, (i.e. meteorological model and MCIP output), with repeated reading and writing of data to/from intermediate files, resulting in reduced data accuracy due to truncation error.

To address these potential shortcomings, we coupled the WRF meteorology model and the CMAQ model to create a two-way coupled modeling system to facilitate feedbacks between chemistry and meteorology. Feedback can be categorized as direct or indirect effect: the former deals with how aerosol affects the radiation and the latter considers how aerosols affects cloud formation and duration resulting from scattering and absorption as well as acts as $\mathrm{CCN}$ which impact cloud optical thickness and cloud lifetime. In this article, we focus on the direct effect. Section 2 provides an overview of the scientific components in the model. Section 3 describes the software considerations for developing a flexible and efficient coupled modeling system, including domain decomposition and design issues. Preliminary results are presented in Sect. 4 while Sect. 5 summarizes the main results and presents a brief discussion of future work. 


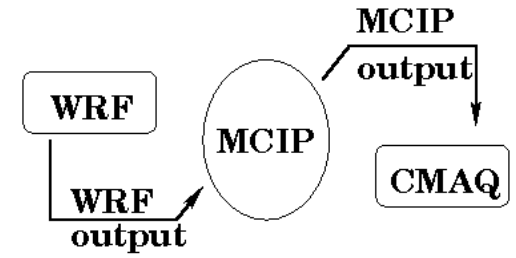

(a)

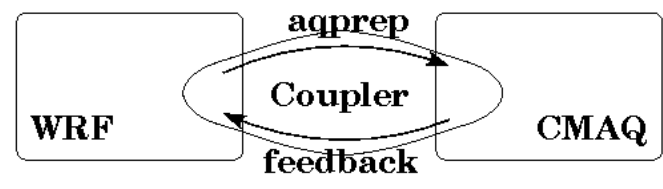

(b)

Fig. 1. Schematic of high-level modules in (a) offline WRF-CMAQ system and (b) two-way coupled 2 WRF-CMAQ model.

\section{Overview of scientific components of the coupled model}

The coupled modeling system consists of three components: the WRF meteorology model, the CMAQ model and the coupler. A high level view of the system is depicted in Fig. 1b. A detail description of each component is provided below.

\subsection{WRF}

The Advanced Research WRF version 3 (WRF-ARW) is a state-of-the science mesoscale meteorology model (Skamarock et al., 2008) that is typically configured with horizontal grid resolutions ranging from $1-30 \mathrm{~km}$, but WRF is also being used on Large Eddy Simulation Scale (dx 100m or smaller) (Moeng, 2007) as well as on global scales. The dynamical equations numerically solved by the WRF-ARW model are fully compressible, Euler nonhydrostatic, and are conservative for all scalar variables. The prognostic variables are the three velocity components, perturbation potential temperature, perturbation geopotential, and perturbation dry air surface pressure. Additional prognostic variables depend on the model physics options and may include turbulent kinetic energy, water vapor mixing ratio, and several cloud microphysical scalars such as cloud water/ice mixing ratio, rain/snow mixing ratio, and graupel mixing ratio. Both the WRF-ARW and the CMAQ model can be configured to use the exact same grid configurations and coordinate systems. Thus, no spatial interpolation of either meteorological or chemical data is required.

\subsection{CMAQ}

CMAQ version 4.7.1 (Foley et al., 2010) is a comprehensive atmospheric chemistry and transport model that numerically integrates a set of independent chemical conservation of mass equations on a series of 3D nested Eulerian grid meshes. The CMAQ model employs operator splitting to modularize the various physical and chemical processes including: subgrid turbulent vertical transport, horizontal and vertical advection, horizontal diffusion, cloud processes (i.e. aqueous chemistry, subgrid convective transport, wet deposition), gas-phase chemistry, and aerosol chemistry and dynamics. The CMAQ system includes anthropogenic emission rates processed by the Sparse Matrix Operator Kernel Emissions (SMOKE) [http://www.cep.unc.edu/ empd/products/smoke]. Plume rise, biogenic emissions and dry deposition are modeled by components of the CMAQ model. Both sources (emissions) and sinks (deposition) are applied as mass tendencies in the vertical diffusion calculation.

\subsection{Coupler}

The coupler is used to link these two models together and serves as an inter-model translator. The design and functionality will be described in the next section. The coupler also includes software (aqprep) to transfer meteorological fields from WRF to CMAQ and to transfer aerosol predictions from CMAQ back to WRF (feedback).

A subroutine called aqprep prepares meteorological fields in forms compatible for use in CMAQ's generalized coordinate formulation. The preparation includes extracting data such as pressure and wind field directly from WRF and calculating additional variables that are used in CMAQ such as the vertical coordinate Jacobian and the fractional area of each land use category in each grid cell. In essence, aqprep includes the functionality currently embodied by the MCIP (Otte and Pleim, 2010) preprocesses in the offline WRF CMAQ system.

An important benefit of two-way coupling between meteorology and air quality models is the ability to use aerosol fields simulated by the air quality model to affect processes in the meteorology model. The first feedback implemented in the WRF-CMAQ system is the direct effects by which chemical species calculated in CMAQ are transferred to WRF for calculating their influence on radiation computed in WRF. In addition to the data coupling described in the next section, implementation of direct feedback requires a new subroutine for the calculation of the aerosol optical properties: extinction optical depth, single scattering albedo, asymmetry parameter, and forward scattering fraction, for short-wave spectral bands (19 bands in Community Atmosphere Model (CAM) and 14 bands in Rapid Radiative Transfer Model for GCMs (RRTMG)). The aerosol 
chemical species calculated by CMAQ are combined into five groups: water-soluble, insoluble, sea-salt, black carbon, and water. The refractive indices for these species are taken from the OPAC (Optical Properties of Aerosols and Clouds) (Hess et al., 1998) database using linear interpolation to the central wavelength of the RRTMG wavelength intervals. These direct feedbacks tend to reduce $\mathrm{SW}$ radiation reaching the ground in areas of high aerosol loading, thereby reducing daytime surface temperatures, as shown in Sect. 4. In addition, absorbing aerosols, such as black carbon, tend to warm the air in layers with high concentrations. There are also secondary effects on PBL heights and cloud properties.

In WRF-Chem (Fast et al., 2006) the optical processes are done by calculating extinction, scattering and asymmetry factor by summing a parametric method originally developed for a modal approach by Ghan et al. (2001). This approach uses first calls a full Mie code to calculate optical properties over exponentially spaced intervals of $\times\left(2^{*} \mathrm{pi} *\right.$ radius/wavelength) for a set of seven refractive indices. A polynomial fit is made for extinction, scattering, and asymmetry factor for each of the refractive indices. All subsequent calls extinction, scattering and asymmetry factor use the polynomial approximations. The integral properties are calculated by summing overall size bins.

In our approach, feedback effects from chemical species calculated by CMAQ are transferred to WRF for calculating the influence of these species on the heat balance computed by WRF. The new aerosol codes to be used with CAM and RRTMG calculate the aerosol extinction, single scattering albedo, and asymmetry factor for short-wave (SW) radiation and aerosol extinction for long-wave (LW) radiation. The aerosol chemical species calculated by CMAQ are combined into five groups: water-soluble, insoluble, sea-salt, black carbon, and water. The refractive indices for these species are taken from the OPAC database using linear interpolation to the central wavelength of the CAM and RRTMG wavelength intervals.

The efficient Gauss-Hermite numerical-quadrature method calculates the extinction and scattering coefficients along with the asymmetry factor by integrating the Bohren \& Huffman Mie codes over the log-normal size distributions representing the Aitken, accumulation, and coarse modes produced by CMAQ.

\section{Software considerations for the coupler}

An air quality model may utilize a different map projection, time integration, grid orientation, grid cell size, and/or vertical coordinate that is different from its meteorological driver. In order to facilitate communication between models to exchange relevant information that is usable by each individual model, a coupler is devised.

\subsection{Modeling domain}

Both the WRF-ARW and CMAQ use the Arakawa C horizontal grid staggering, and in the coupler no spatial interpolation of meteorological or chemical data is required. In addition, both WRF and CMAQ use the same map projection, so the coupler inherits the map projection from WRF. The vertical coordinate in WRF is a hydrostatic sigma-pressure, and CMAQ uses a modified, generalized form of that coordinate. Unlike in the offline WRF-CMAQ system, the coupler must use the same number and configuration of vertical layers (i.e., no layer collapsing, Otte and Pleim, 2010). Figure 2 illustrates the typical domain configurations of the WRFCMAQ coupled system; in this the chemistry-transport calculations are performed for a sub-domain of the larger WRF domain. In typical WRF applications, to provide a transition from externally specified lateral boundary conditions, a relaxation zone is specified where the model is relaxed toward the large-scale forecast (Skamarock et al., 2008). The coupler allows the users to specify how many grid cells to trim off for the chemistry-transport calculations at run time, but five grid cells is the recommended minimum to avoid numerical artifacts that commonly occur in the WRF boundary relaxation zone. Horizontal transport calculations in CMAQ only require the specification of species concentrations (time varying or independent based on user specification) at a onecell thick boundary along the CMAQ domain. Thus, under conditions of inflow, the concentrations specified at these upwind boundary cells are used to estimate the advective flux (following the PPM formulation) into the domain. Users can define any CMAQ domain as long as that domain fits within the maximum CMAQ domain depicted in Fig. 2. In addition, the user is required to provide the value of delta_X and delta_y which defines the lower left corner of the CMAQ domain relative to the WRF domain. Because the WRF model is the driver and CMAQ is called as a subroutine within WRF, a global timer that is based on the WRF advection time step is used to synchronize WRF and CMAQ in the coupled system.

\subsection{Domain decomposition}

The main task of the coupler is to transfer needed data between these two models correctly. Both models were designed to run in a parallel computing environment. WRF supports MPI and OpenMP but the current version of CMAQ only supports MPI. They both use domain decomposition as the basic parallelization approach. However, the details of the decomposition are quite different in both models. Runtime System Library (RSL) (Michalakes, 1994) and RSL-lite (Michalakes, 1998), which both handle high-level stencil and inter-domain communication, irregular domain decomposition, automatic local/global index translation, distributed I/O, and dynamic load balancing, are used in WRF to parallelize the code. RSL-lite is a bit faster than RSL. Both RSL and RSL-lite were used in WRF version-2 implementation, but RSL has been removed since version 3 . 


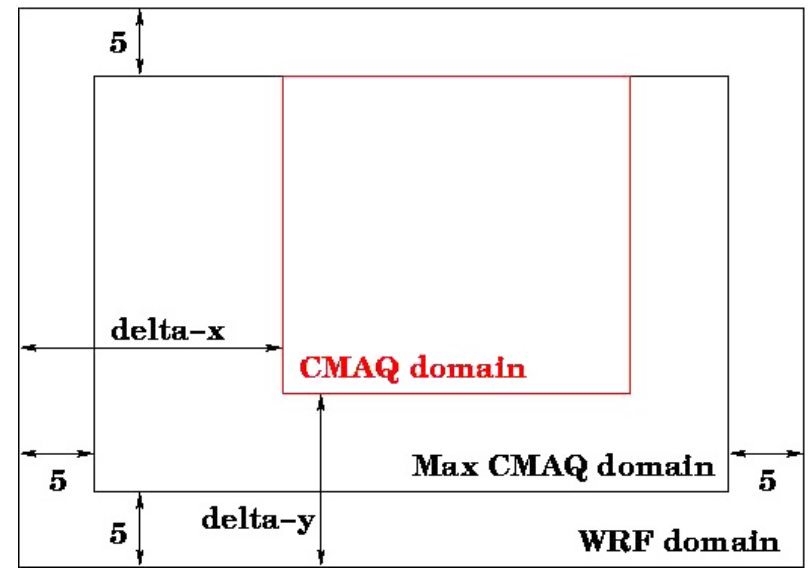

Fig. 2. WRF and CMAQ domain orientation in coupled system.

Besides performance, the main difference between RSL and RSL-lite is the domain decomposition algorithm. Since RSL has been removed, the description of the decomposition algorithm is focused on RSL-lite. The mapping between processor and sub domain is in row-wise fashion. The starting point and order of assigning the remainder row elements is at the bottom and then top, and then bottom until all the remaining elements are distributed (Fig. 3a). Similarly, for the column dimension, it starts at the left and then right and moving towards the center.

In CMAQ, the domain decomposition uses the same processor and sub-domain mapping as in WRF (Fig. 3b), but the starting point and order of assigning the remainder column or row elements is different. The remainder elements starts from the bottom and moves toward the top for row dimension and starts from left and moves toward the right for column dimension, rather than alternating inward from the top/bottom and left/right.

When CMAQ is executed, users can choose a particular processor configuration based upon the number of processors allocated. For instance, if the number of available processors is 16 , the user can choose between a $4 \times 4,8 \times 2$, $2 \times 8,16 \times 1$ or $1 \times 16$ processor configuration. In WRF, the processor configuration with a "square" orientation, is the default but it is user-definable. In the WRF-CMAQ coupled system, CMAQ's processor configuration is inherited from WRF. The coupler's main task is to compute the mapping between the WRF and CMAQ domains with respect to each sub domain with consideration of the position of the CMAQ domain relative to the WRF domain. This mapping information will be used for data transfer between these two models in the forward and feedback steps.

\subsection{Data exchange}

We have considered different tools, such as ESMF (Earth System Modeling Framework) (http://www. earthsystemmodeling.org/), Cpl6 (Craig et al., 2005) and MCT (Larson et al., 2005), for data exchange between the two models. CMAQ uses the IOAPI3 (Input/Output Applications Programming Interface version 3, http://www.baronams.com/products/ioapi/AVAIL.html) to handle physical file $\mathrm{I} / \mathrm{O}$. With the consideration of minimal code change, we chose to use IOAPI3 for the coupling. The actual data transfer is performed in memory through IOAPI3 buffered files. IOAPI3 is third party software written by Baron Advanced Meteorological Systems (BAMS). It is written to handle various types of files: volatile real files which are used in CMAQ to deal with I/O, using the netCDF format; buffered virtual files which facilitate data exchange within the same program through memory; coupling-mode virtual files which use the PVM3.4 mailbox mechanism to exchange data among models executed concurrently; and native-binary real files which are the same as the volatile real files except the file is stored in native binary format instead of netCDF.

The type of file, whether it is a volatile real file or a buffered virtual file, used in the application is determined at run time. The capability of handling various file types within IOAPI3 is transparent to the user application code; hence, code modification is not needed. The coupler will create/open the same number of files as in the offline run that uses physical files. In the stand-alone CMAQ model, each processor is able to access the entire file and only extract relevant data for the sub-domain portion from the file. In addition, hourly meteorological input is interpolated to the current time step in various science processes within CMAQ throughout the execution. In order to make the same code work for buffered files while reducing memory consumption, each buffered file is exactly the same size as a sub-domain and corresponds to that sub-domain only. Two time steps of data are stored in each circular buffered file.

\subsection{System structure}

The coupler consists of two major components: aqprep and feedback. The prepared data is placed in the corresponding buffered files which have similar attributes as the physical files used in the uncoupled stand-alone CMAQ. In CMAQ, the same IOAPI 3 calling interface as in the stand alone model is used to access these buffered files. This design provides flexibility to read and write either buffered or disk files, enabling consistent coupled and uncoupled modeling paradigm. Thus an inherent advantage of using the IOAPI3 to handle the file format in the coupler is that minimal code changes are needed in CMAQ. The feedback part is called within the aerosol module in CMAQ and computes several variables that are needed for direct aerosol feedback to the 


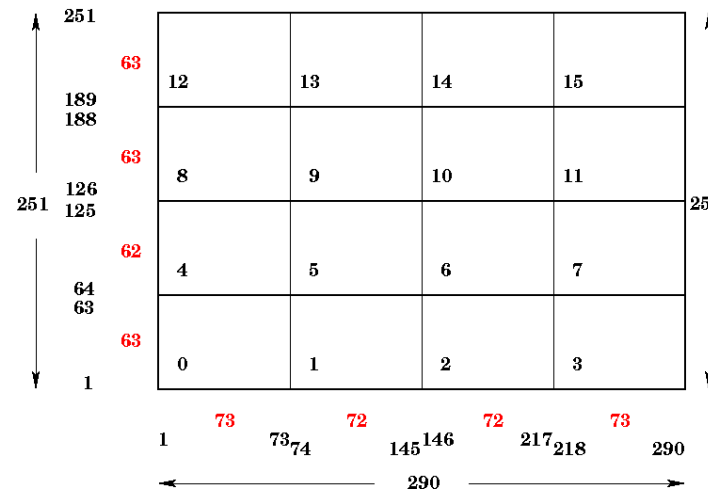

(a)

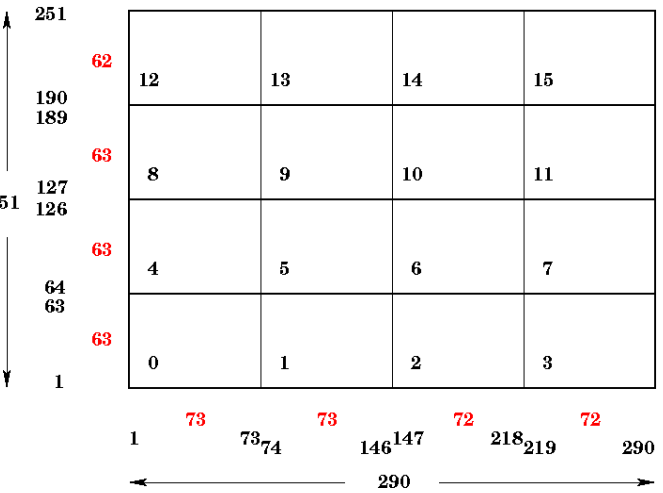

(b)

Fig. 3. Decomposition with a $4 \times 4$ processor configuration for RSL-lite (a) and CMAQ (b).

WRF radiation module. Various information such as coarse mode diameter and Aitken mode natural log of standard deviation, is used to compute soluble mass, elementary carbon mass and other parameters.

WRF integrates at a very fine time step, e.g. one minute for $12 \mathrm{~km}$ horizontal grid cell size. In CMAQ each physical process, (e.g. transport and chemistry), has a different time step requirement that is based on individual process characteristic time scales and numerical stability criteria. As a stand-alone model, CMAQ determines the minimum synchronization time step based on the horizontal wind speed Courant condition in model layers lower than $\sim 700 \mathrm{hPa}$ which generally allows for CMAQ's synchronization time step to be several times greater than the WRF time step. In coupled system, users can choose the call frequency at run time as a ratio between the WRF and CMAQ time steps. For instance, if the ratio is set to four and the WRF time step is $30 \mathrm{~s}$, the CMAQ time step will be two minutes. Consequently, the computational burden for the coupled system increases substantially as the CMAQ calling frequency increases. The non-linear increase in computational intensity is related to inherent nonlinearity in atmospheric processes and numerical solution of the governing equations. Figure 4 depicts the calling sequence for the coupled system. In general, CMAQ is called after an aqprep step except the very first time. This implementation ensures two steps of WRF data are always available in case temporal interpolation of meteorological information is needed in CMAQ. The feedback step takes place in CMAQ within the aerosol module. Since CMAQ is a subroutine of WRF it occupies a portion of a WRF step. There are two versions of the radiation calculation within the two-way coupling model: one comes with the WRF code (Ra) and the other was modified to include feedback capability (Ra'). Ra' is not called until feedback information is available. That is why $\mathrm{Ra}$ is used for the very first step.

\subsection{Software modification in WRF and CMAQ to support the coupled system}

In the coupled system, CMAQ is implemented as a subroutine in WRF. Since CMAQ is a community model with a wide user community which has used the model in an offline mode, all the coupling related functions are encapsulated in Fortran 90 modules that will not be invoked when running CMAQ in a stand-alone offline mode. Thus, the same version of CMAQ can be consistently used both in an offline or coupled system mode. The remainder of the necessary modifications to CMAQ to enable online coupling are transparent to the user. Whether to include the coupling portion or not in CMAQ is decided at compilation time. This fulfills the single source design principle and eliminates software maintenance of separate model versions for on-line and off-line configurations.

The aerosol optical depth for nine selected wavelength bands estimated from the simulated aerosol distribution is added to the WRF output for examination and validation purposes. This requires nine new variables in the WRF Registry. Two routines in WRF were also modified for the coupled system: solve_em.F (to invoke CMAQ) and the radiation calculations (to add the nine variables which are passed into various parts of the radiation calculation and to process aerosol feedback). These modifications can be easily ported to newer versions of WRF as its science is updated.

Run time switches have been implemented to disable the aerosol feedback in the coupled system as well as to run WRF in stand-alone mode (i.e., without calling CMAQ). These options provide flexibility to perform sensitivity studies on the effects of the feedback mechanism and the coupling. 


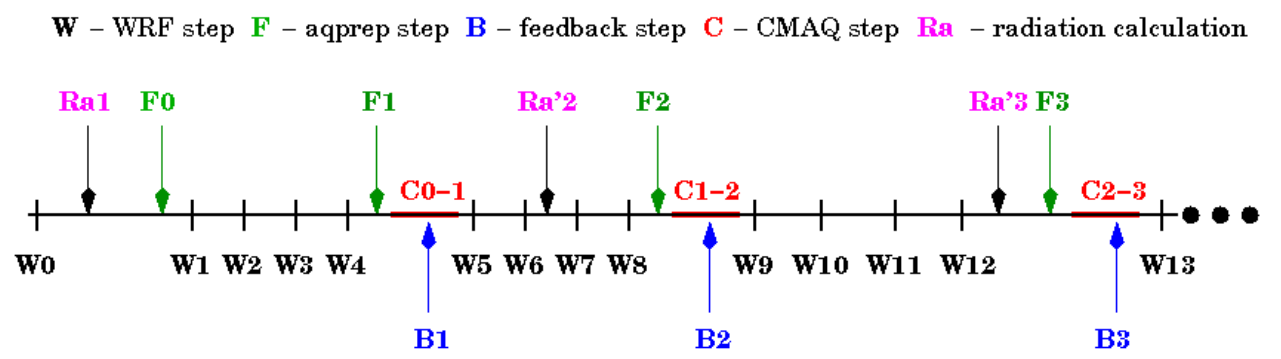

Fig. 4. Calling sequence of the coupled system with $4: 1$ call frequency and radiation is updated every 6 time steps.

\section{Preliminary performance of the coupled system}

The performance of the WRF-CMAQ two-way coupled system is evaluated for both computational impact and scientific advancement. In order to be of general use to the CMAQ community, the coupled system must not add such a computational burden that it would become prohibitive for the average user to run. In addition, it is desirable that the coupled system scales well computationally as processors are added to the configuration. Of equal importance is the need for the coupled system to demonstrate a scientific advantage beyond what could be achieved by using the WRF and CMAQ models sequentially.

\subsection{Computational performance}

We conducted a series one-day simulation (1 August 2006) to examine and quantify the computational performance of the coupled system. For these tests, we used a domain encompassing Eastern US, discretized with 12-km horizontal grid spacing while the vertical extent ranging from the surface to $50 \mathrm{hPa}$ was discretized using 34 layers of variable thickness. Here, the WRF domain size is $290 \times 251$ grid points, and the CMAQ domain size is $279 \times 240$ grid cells, which allows for the five-cell boundary along the perimeter of the WRF domain to be excluded from CMAQ (Fig. 2). WRF-ARW version 3.1 was built with CMAQ version 4.7.1 to form the WRF-CMAQ two-way coupled system. Initial and lateral boundary conditions for WRF were derived from a combination of North American Mesoscale (NAM) model analyses and forecasts at $3 \mathrm{~h}$ intervals that were developed by the National Center for Environmental Prediction and obtained from the National Climatic Data Center. In WRF, the model options included the WRF single-moment 6-class (WSM6) microphysics scheme (Hong et al., 2004), version 2 of the Kain-Fritsch (KF2) cumulus cloud parameterization (Kain, 2004), the Asymmetric Convective Model version 2 (ACM2) for the planetary boundary layer (Pleim, 2007a, b), and the Pleim-Xiu land-surface model (Xiu and Pleim, 2001) with soil moisture and temperature nudging (Pleim and Xiu, 2003; Pleim and Gilliam, 2009). Both the Rapid Radiative Transfer Model for GCMs (RRTMG) (Clough et al., 2005) and Community Atmospheric Model (CAM) (Collins et al.,
2004) radiation schemes were tested and run in the coupled system to contrast the simulated impact of radiative feedback from CMAQ to WRF using multiple radiation schemes. Also in WRF, analysis nudging was included for temperature and humidity above the PBL and for winds at all model levels (Stauffer et al., 1991).

In CMAQ, the CB05 gas-phase chemical mechanism and the modal aerosol model known as AERO5 (Carlton et al., 2010) were used. The same subgrid vertical turbulent transport of meteorological and chemical species was used in both WRF and CMAQ following the ACM2 PBL scheme. The sub-grid convective cloud scheme in CMAQ, which is responsible for convective transport of chemical species, aqueous chemistry, and wet scavenging, is a simple bulk scheme based on the convective cloud model in the Regional Acid Deposition Model (RADM; Chang et al., 1987) but with convective transport based on the Asymmetric Convective Model (Pleim and Chang, 1992). Since the CMAQ cloud scheme uses the convective precipitation rate to diagnose sub-grid mass fluxes, the location and timing of precipitating convective clouds are consistent with WRF. A new convective cloud scheme based on the Grell scheme in WRF (Grell and Devenyi, 2002) is being tested to improve consistency across chemical and meteorological components of the system. Note that WRF and CMAQ use different scalar advection schemes that are both monotonic and positive definite for meteorological and chemical species. However, differences in numerical formulations and time steps allow subtile differences in the 3-d mass fields to accumulate over time. Mass conservation and consistency between chemical concentrations and air density is ensured in CMAQ by adjustment of the vertical velocities according to a layer-by-layer solution of the 3-d mass continuity equation at every time step. In this design chemical species are advected in CMAQ by an efficient scheme that has very little numerical diffusion: the piecewise parabolic method (PPM) (Colella and Woodward 1984). A potential drawback of this approach is the inconsistencies between advective transport of microphysical scalars in WRF and advection of gas and aerosol species in CMAQ. While such discrepancies are likely very small they could be important for modeling aerosol indirect effects which result from interactions between aerosols and cloud microphysics. 
The significance of these inconsistencies will be assessed as implementation and testing of indirect aerosol processes continues.

For these tests, a one-minute WRF time step was used, and CMAQ was called every five WRF time steps (ratio of 5:1). The simulations were run on 32 processors on a Linux cluster.

Table 1 presents the execution time of the offline WRFCMAQ system, the WRF-CMAQ two-way coupled system with and without radiative feedback. When the coupled system is executed without radiative feedback (but with increasing the temporal frequency of the WRF meteorological fields available for CMAQ), computational time is virtually identical when using the CAM radiation option and a slightly increased $(\sim 8.5 \%)$ when using the RRTMG radiation option compared to the offline WRF-CMAQ system. Once the feedback mechanism is turned on, the execution time increases only slightly with CAM but increases about $60 \%$ with RRTMG. The numerical techniques used to compute aerosol optical characteristics (extinction, scattering, and asymmetry factor) with the Mie approach used in RRTMG are much more computationally intensive than the Mie approximation (Evans and Fournier, 1990) used in the CAM implementation. However, this new scheme used for RRTMG is more accurate and robust over a wider range of refractive indices.

The individual models used in the coupled system, WRF and CMAQ, are fully parallelized. As a result, the scalability of the coupled system is inherited from both components. By doubling the processors from 32 to 64 on this domain, a speedup of $\sim 1.6$ was achieved for both CAM and RRTMG configurations, regardless of whether the radiative feedback was enabled (Table 2). Increasing the number of processors by a factor of four (32 to 128) resulted in a speedup of $\sim 2.3-$ 2.7. The addition of radiative feedback in the coupled system does not adversely affect the scalability using either CAM or RRTMG, but results in a greater relative speedup on more processors.

\subsection{Scientific performance}

To examine the scientific performance of the coupled system with radiative feedback, we conducted a ten-day simulation (20 June - 29 June 2008) of a wildfire event in California. Widespread wildfires (Fig. 5) resulted in significant particulate matter (PM) pollution during mid-late June 2008 in California and surrounding states. The coupled model using the options discussed in Sect. 4.1, was applied to a domain which covers California and portion of the surroundings states (Fig. 6); the RRTMG radiation scheme was used and the vertical extent up to $100 \mathrm{mb}$ was discretized using 22 layers. This simulation uses the latest fire emission data (ftp://ftp.epa.gov/EmisInventory/2005v4/2007emis/ smartfire_and_bluesky_enabled_methodology_2006_2008.

pdf). Model results from simulations with and without radiative feedback were compared to ground-level meteorology

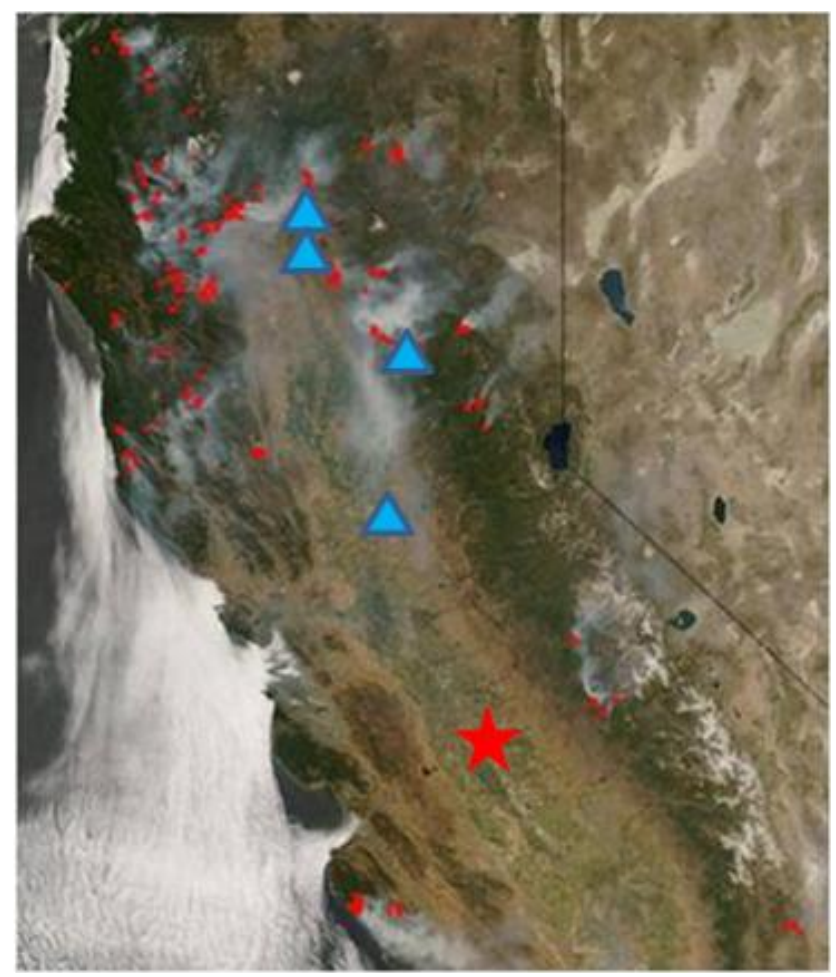

Fig. 5. Locations of the June 2008 California wildfires (captured by NASA.s Aqua Satellite, http://www.nasa.gov/topics/earth/features/ fire_and_smoke.html ) illustrated by the red dots. Also shown are the locations of observations sites whose data is shown in Figs. 7 and 8 .

measurements from the Meteorological Assimilation Data Ingest System (MADIS), radiation measurements from the Integrated Surface Irradiance Study (ISIS) Network, and concentration measurements from the US Environmental Protection Agency's Air Quality System (AQS) network.

Figure 6 presents an illustration of the direct aerosol feedbacks simulated by the coupled WRF-CMAQ modeling system during the time period of these wild fires in California. In general relatively high aerosol optical depths are noted in regions of high surface and boundary-layer particulate matter pollution (Fig. 6a and b). Shown in Fig. 6c is the difference in surface shortwave radiation between a run with aerosol feedback and one without, while Fig. 6d presents an illustration of a similar difference in the modeled planetary boundary layer (PBL) height at the same time. As illustrated, aerosol direct radiative effects associated with scattering and absorption of incoming radiation, result in a reduction of short-wave radiation reaching the surface (Fig. 6c), which then translate to reduction in temperature at the surface as well as a reduction in PBL height (Fig. 6d). These effects are particularly pronounced in regions with high aerosol loading with simulated reductions of over $250 \mathrm{Wm}^{-2}$ in instantaneous surface shortwave radiation and corresponding reductions of over $500 \mathrm{~m}$ in PBL heights. 
Table 1. Performance of the uncoupled and coupled system (hh:mm:ss) on a linux cluster.

\begin{tabular}{lrr}
\hline & \multicolumn{2}{r}{ Execution time } \\
\cline { 2 - 3 } & CAM & RRTMG \\
\hline WRF only & $0: 19: 59$ & $0: 18: 50$ \\
MCIP & $0: 02: 31$ & $0: 02: 31$ \\
Offline CMAQ & $1: 18: 28$ & $1: 19: 05$ \\
Offline WRF-CMAQ system, Total time & $1: 40: 58$ & $1: 40: 26$ \\
\hline Coupling system w/o feedback and call frequency ratio 5:1 & $1: 41: 12$ & $1: 48: 59$ \\
\hline Coupling system w/ feedback and call frequency ratio 5:1 & $1: 43: 39$ & $2: 54: 25$ \\
\hline
\end{tabular}

Table 2. Computational performance (hh:mm:ss) and scalability on a linux cluster.

\begin{tabular}{lllllllll}
\hline $\begin{array}{l}\text { Processor } \\
\text { configuration }\end{array}$ & CAM & & & & RRTMG & & \\
& w/o feedback & speedup & w/ feedback & speedup & w/o feedback & Speedup & w/ feedback & speedup \\
\hline $4 \times 8$ & $2: 05: 06$ & & $2: 08: 21$ & & $2: 13: 17$ & & $3: 19: 25$ \\
$8 \times 8$ & $1: 19: 46$ & 1.57 & $1: 21: 57$ & 1.57 & $1: 24: 12$ & 1.58 & $1: 58: 21$ & 1.68 \\
$8 \times 16$ & $0: 55: 28$ & 2.26 & $0: 55: 12$ & 2.33 & $0: 56: 38$ & 2.35 & $1: 14: 14$ & 2.69 \\
\hline
\end{tabular}

Figure 7 presents comparisons of the surface shortwave radiation simulated for the cases with and without direct aerosol feedback with measurements at an Integrated Surface Irradiance Study (ISIS) site in Hanford, California (see Fig. 5, red star). The observations show that there was significant reduction of incident shortwave radiation at the surface (peak observed values of $900-1000 \mathrm{Wm}^{-2}$ ) on 24 , 26 and 27 June due to the smoke plumes from the wildfires. Without aerosol feedback effects, the model overestimates shortwave radiation by $\sim 100-200 \mathrm{Wm}^{-2}$. When the aerosol feedbacks were included, the model bias was significantly reduced, though a slight overestimation still persists. Thus including the aerosol feedback in the coupled system is important for better simulating the shortwave radiation fields in WRF.

The presence of aerosols from the wildfires reduces the shortwave radiation at the surface, which also acts to reduce the maximum daytime temperatures near the surface. Figure 8 shows a comparison of 2-m temperatures averaged from four sites in the Sacramento Valley, (Oroville, Red Bluff, Redding, and Sacramento, see blue triangles in Fig. 5) with model simulations for the cases with and without radiation feedback, for the ten-day simulation period. Typically the 2-m temperature was overestimated in the simulation that did not consider any aerosol feedback effects. This overestimation was reduced in the simulation with the aerosol feedbacks. For example, on 25, 26, 27 and 29 June, when the wildfires were most actively affecting these sites, inclusion of the aerosol feedback reduced or eliminated the persistent over prediction evident in the simulation without feedback.

Figure 9 presents comparisons of the day time $(8 \mathrm{a} . \mathrm{m}$. to 6 p.m. local time) model and observed ambient levels of ozone and $\mathrm{PM}_{2.5}$ for all sites and data pairs; model results for both simulations with and without the feedback effects are shown. Figure 10 presents similar comparisons but only for data pairs where the simulated $\mathrm{AOD}>0.5$. While somewhat arbitrary this criteria helps examine the model performance for cases of significant aerosol loading, and consequently wherein radiative feedback effects on temperature and PBL heights could in turn influence the subsequent chemistrytransport simulation. Though modest, the simulation including the aerosol feedback effects exhibits slight higher correlation coefficients than the one without.

\section{Summary and future work}

A two-way coupled meteorological and air quality modeling system has been developed by linking the WRF and CMAQ models. The system represents advancement over the traditional offline WRF-CMAQ system because the aerosols predicted by CMAQ are able to impact the clouds, radiation, and precipitation simulated by WRF in a consistent online coupled manner. In addition, because CMAQ is called directly from WRF, the temporal interpolation of meteorological fields from WRF is eliminated thereby improving consistency in the use of meteorological information in the chemistry-transport calculations. A coupler is developed to efficiently link the two model systems. The 


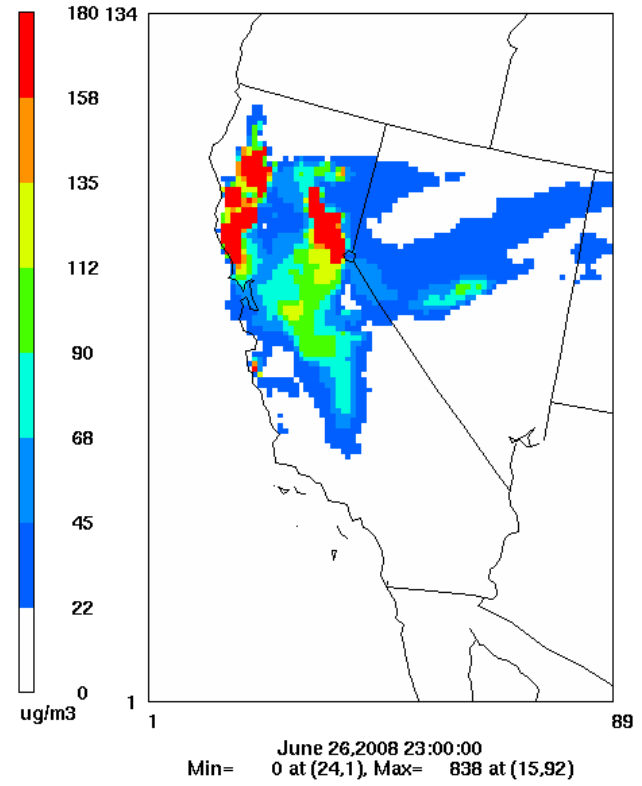

(a) surface $\mathrm{PM}_{25}\left(\mu \mathrm{g} \mathrm{m}^{-3}\right)$

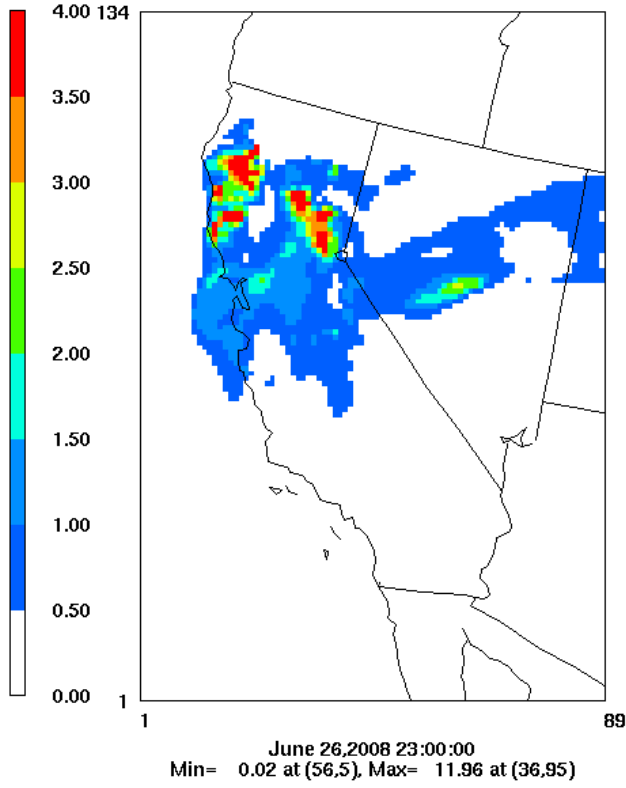

(b) AOD

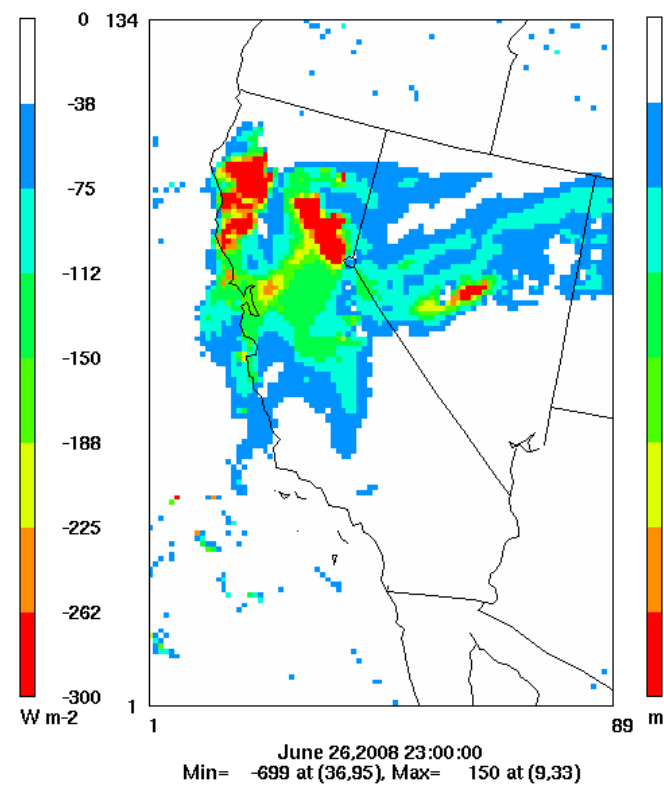

(c) reduction in $\mathrm{SW}\left(\mathrm{Wm}^{-2}\right)$

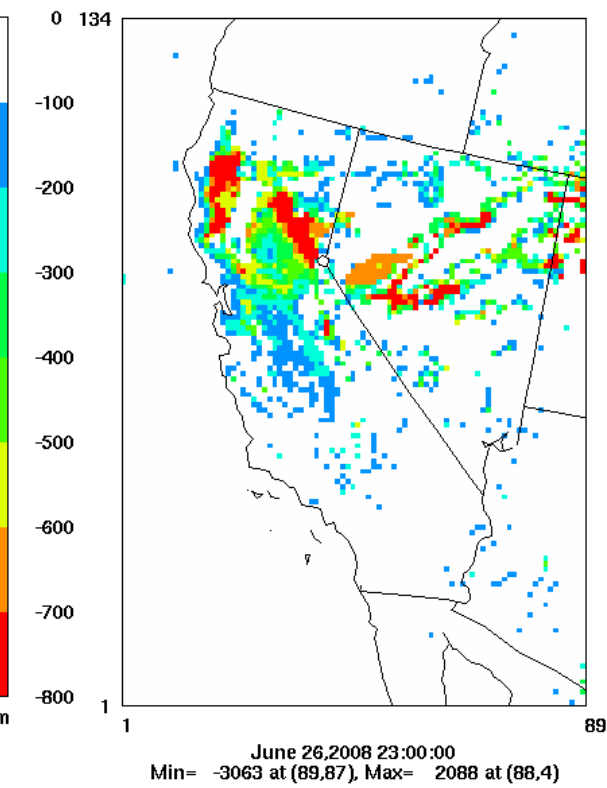

(d) reduction in PBL (m)

Fig. 6. Illustration of the direct radiative effects of aerosols simulated by the coupled WRF-CMAQ model at 22:00 UTC on 25 June 2008.

coupler handles communication between WRF and CMAQ, performs translation of the WRF fields to drive CMAQ, and provides aerosols feedback information from CMAQ to the WRF. The coupler is encapsulated in Fortran 90 modules so the details of the two-way coupling are transparent to the users. This software design also enables WRF and CMAQ to be detached and executed as stand-alone models as in the traditional offline paradigm. The single-source coding approach minimizes software maintenance so scientific updates to both WRF and CMAQ can be readily incorporated into the coupled WRF-CMAQ system.

In addition to scientific and software maintenance issues, the coupled modeling system was designed to maximize user flexibility for research and applications by imposing 

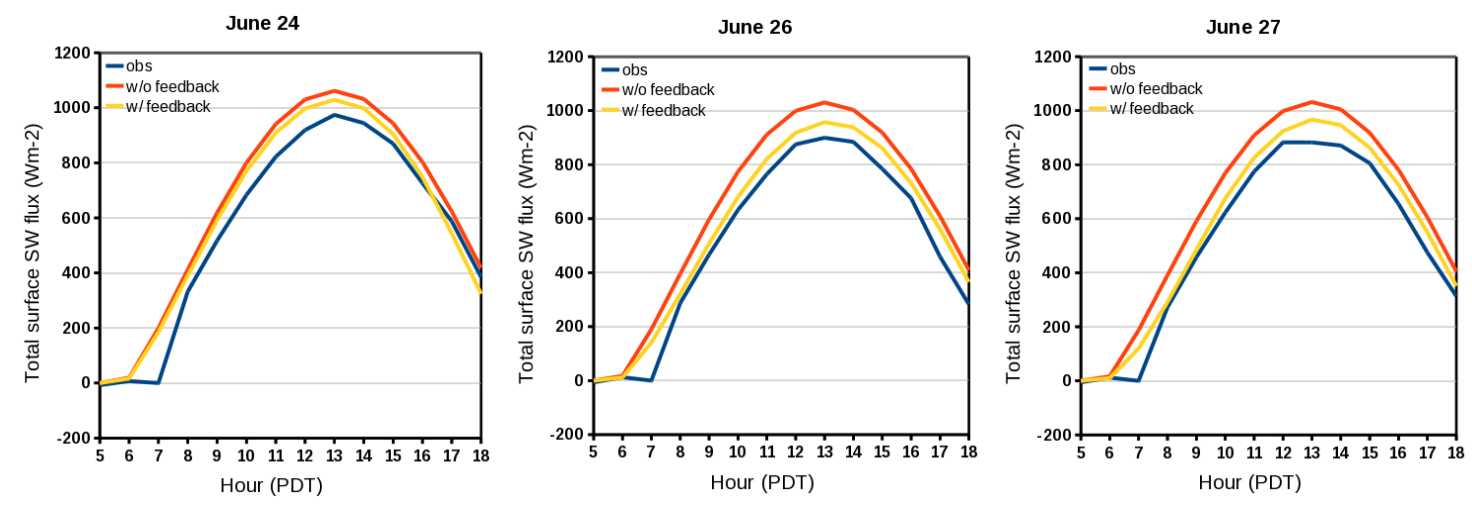

Fig. 7. Total surface short wave radiation comparison between measurements at Hanford, CA (blue), and with (yellow) and without (red) direct aerosol feedback

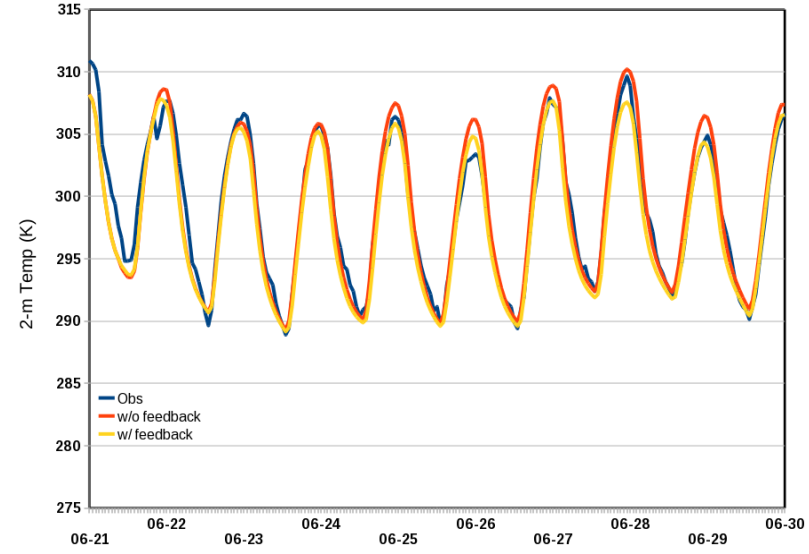

Fig. 8. Surface (2-m) temperature comparison of averaged measurements (blue) from four sites (Oroville, Red Bluff, Redding and Sacramento) with model simulation with (yellow) and without (red) direct aerosol feedback.

minimal restrictions on domain specifications and physics options in both WRF and CMAQ. Furthermore, the coupler allows users to choose the call frequency of CMAQ to balance the computational burden against the scientific accuracy, depending on the availability of computational resources. The coupled modeling system also includes a runtime switch to disable aerosol feedback and emulate the traditional offline paradigm albeit with greater frequency of communication of meteorological information from WRF to the CMAQ model; this option can be used for further sensitivity tests examining the potential effects of temporal interpolation of meteorological data in the traditional offline paradigm.
When aerosol feedback is disabled, the computational time for of the coupled model is virtually identical to the offline WRF-CMAQ system. When the radiative feedback is enabled, there is slight increase in execution time (compared to the case without feedback) using the CAM radiation scheme. However, adding radiative feedback with the RRTMG scheme results in an increase in run time of about $60 \%$, which is largely attributed to the more computationally intensive Mie calculation used in the implementation of the feedback effects with the RRTM scheme. Improving the computational efficiency of the more accurate Mie scheme and its coupling with the RRTM is currently being investigated. In general, the coupled WRF-CMAQ modeling system scales well as the number of processors increase, regardless of the radiation model chosen in WRF or whether or not simulation of feedback effects is enabled.

To demonstrate the improvements in simulated atmospheric dynamical and chemical features with the inclusion of aerosol radiative effects, we conducted a ten-day simulation of a wildfire event in California, a case characterized by significant tropospheric aerosol loading. Including radiative feedbacks in the model noticeably reduced the bias in simulated surface shortwave radiation and 2-m temperatures as well improved the correlation of simulated ambient ozone and $\mathrm{PM}_{2.5}$ relative to observed values. This preliminary analysis suggests that for cases with high aerosol loading (such as from wildfires, or in regions with significant anthropogenic pollution), including the radiative effects of aerosols improves the accuracy of both the meteorology and air quality simulations.

Further model evaluation studies are continuing, including efforts to examine the direct aerosol effects using a closed set of aerosol and radiation observations from the DOE/ARM Southern Great Plains site. Ozone has absorption bands in the long wave radiation bands and can thus absorb outgoing radiation. Efforts are underway to implement ozone feedback in the coupled WRF-CMAQ system and study the impact of ozone on long wave radiation using the RRTMG long 


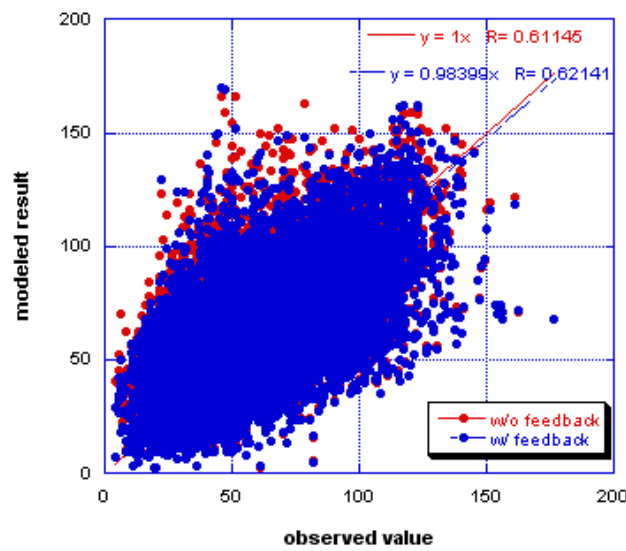

(a) $\mathrm{O}_{3}(\mathrm{ppb})$

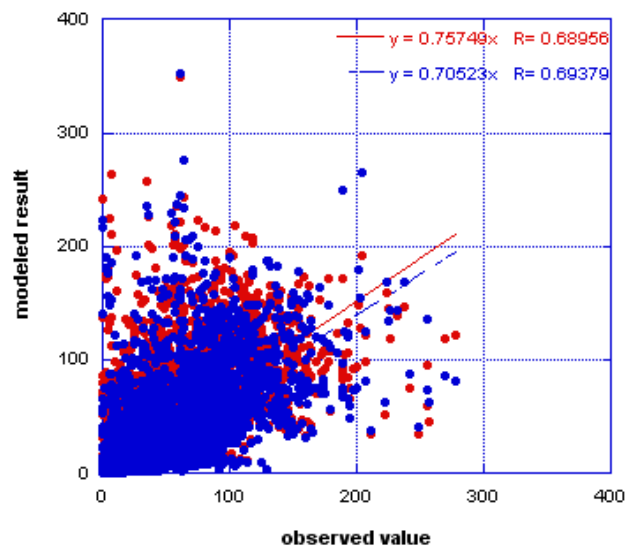

(b) $\mathrm{PM}_{25}\left(\mu \mathrm{g} \mathrm{m}^{-3}\right)$

Fig. 9. Scatter plots of model, with (blue) and without (red) feedback, and observed daytime (a) $\mathrm{O}_{3}$ and (b) $\mathrm{PM}_{2.5}$ ambient levels. Also shown are the slope and correlation coefficient $(R)$.

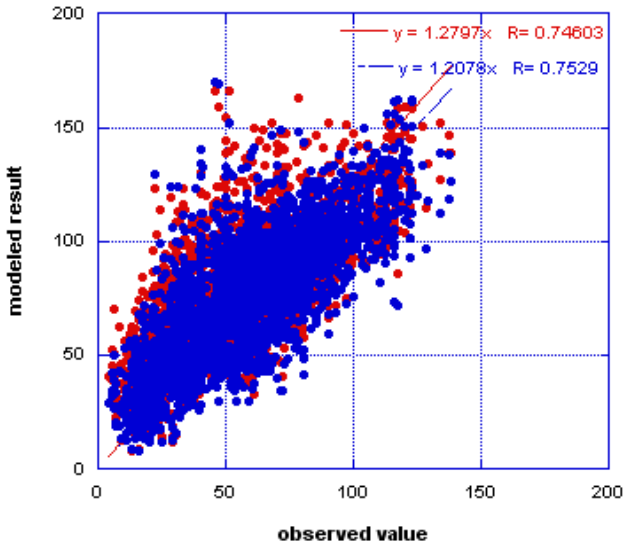

(a) $\mathrm{O}_{3}(\mathrm{ppb})$

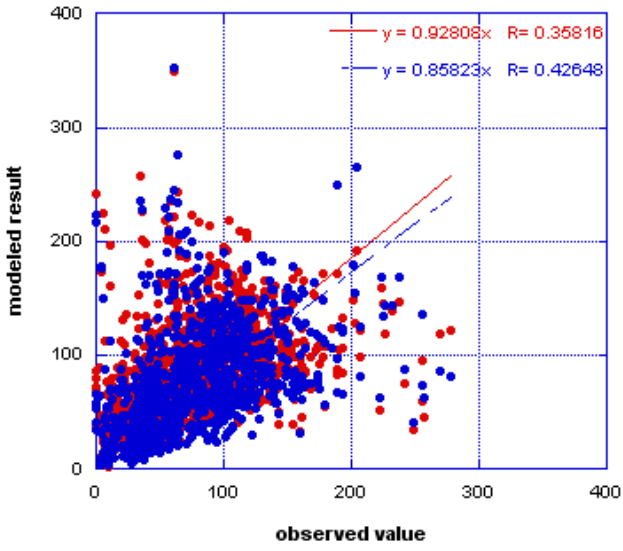

(b) $\mathrm{PM}_{25}\left(\mu \mathrm{g} \mathrm{m}{ }^{-3}\right)$

Fig. 10. Daytime $\mathrm{O}_{3}$ and $\mathrm{PM}_{2.5}$ model performance with $\mathrm{AOD}$ greater than or equal to 0.5 (same colour code as in Fig. 9).

wave radiation scheme. An initial implementation of indirect aerosol forcing has also recently been completed and is under further testing and evaluation. To simulate the most realistic representation of the dynamical state of the atmosphere, four dimensional data assimilation (FDDA) is often employed in retrospective WRF applications. Depending on the strength of the nudging coefficients used, FDDA could dampen the effects of radiative feedbacks in the simulations and such effects need to be further quantified and understood. The overall CPU usage of the coupled WRF-CMAQ system is equivalent to the total CPU time associated with running the offline WRF-MCIP-CMAQ modeling system. A speedup of 2.3 when going from 32 to 128 processors for the Eastern US domain, however, suggests that there is room for further improvement in the parallel performance of the WRF-CMAQ system. Part of this performance hit is due to the input and output files (about ten each) as well as intermediate run time diagnostics. Improvements to the $\mathrm{I} / \mathrm{O}$ in the modeling system are currently under investigation and should result in overall better performance scaling. Improvements in many of these aspects are currently being investigated and will be made available in future versions of the 2-way coupled WRFCMAQ modeling system.

Acknowledgements. The United States Environmental Protection Agency through its Office of Research and Development funded and managed the research described here. It has been subjected to the Agency's administrative review and approved for publication.

Edited by: O. Boucher 


\section{References}

Byun, D. W. and Schere, K. L., Review of the governing equations, computational algorithms, and other components of the Models3 Community Multiscale Air Quality (CMAQ) Modeling System, Appl. Mech. Rev., 59, 51-77, 2006.

Carlton, A. G., Bhave, P. V., Napelenok, S. L., Edney, E. O., Sarwar, G., Pinder, R. W., Pouliot, G. A., and Houyoux, M.: Model representation of secondary organic aerosol in CMAQv4.7., Environ. Sci. Technol., 44, 8553-8560, 2010.

Chang, J. S., Brost, R. A., Isaksen, I. S. A., Madronich, S., Middleton, P., Stockwell, W. R., and Walcek, C. J.: A three-dimensional Eulerian acid deposition model: Physical concepts and formulation, J. Geophy. Res., 92, 14681-14700, 1987.

Clough, S. A., Shephard, M. W., Mlawer, E. J., Delamere, J. S., Iacono, M. J., Cady-Pereira, K., Boukabara, S., and Brown, P. D., Atmospheric radiative transfer modeling: a summary of the AER codes, J. Quant. Spectrosc. Ra., 91, 233-244, 2005.

Colella, P. and Woodward, P. R. The piecewise parabolic method (PPM) for gas-dynamical simulations, J. Comp. Phys., 54, 174201, 1984.

Collins, W. D., Rasch, P. J., Boville, B. A., Hack, J. J., McCaa, J. R., Williamson, D. L., Kiehl, J. T., Bitz, C., Lin, S. J., Zhang, M., and Dai, Y.: Description of the NCAR Community Atmosphere Model (CAM3.0), National Center for Atmospheric Research Tech. Note, NCAR/TN-464+STR, 226 pp., 2004.

Craig, A. P., Jacob, R. L., Kauffman, B., Bettge, T., Larson, J., Ong, E., Ding, C., and He, Y., CPL6: The new extensible, high performance parallel coupler for the Community Climate System Model, Int. J. High Perf. Comput. Appl., 19, 309-327, 2005.

Evans, B. T. N. and Fournier, G. R.: Simple approximation to extinction efficiency valid over all size parameters, Appl. Optics, 29, 4666-4670, 1990.

Fast, J. D, Gustafson, Jr., W. I., Easter, R. C., Zaveri, R. A., Barnard, J. C., Chapman, E. G., and Grell, G. A.: Evolution of ozone, particulates, and aerosol direct forcing in an urban area using a new fully-coupled meteorology, chemistry, and aerosol model, J. Geophys. Res., 111, D21305, doi:10.1029/2005JD006721, 2006.

Foley, K. M., Roselle, S. J., Appel, K. W., Bhave, P. V., Pleim, J. E., Otte, T. L., Mathur, R., Sarwar, G., Young, J. O., Gilliam, R. C., Nolte, C. G., Kelly, J. T., Gilliland, A. B., and Bash, J. O.: Incremental testing of the Community Multiscale Air Quality (CMAQ) modeling system version 4.7, Geosci. Model Dev., 3, 205-226, doi:10.5194/gmd-3-205-2010, 2010.

Ghan, S. J., Laulainen, N. S., Easter, R. C., Wagener, R., Nemesure, S., Chapman, E. G., Zhang, Y., and Leung, L. R.: Evaluation of aerosol direct radiative forcing in MIRAGE, J. Geophys. Res., 106, 5295-5316, 2001.

Grell, G. A. and Baklanov, A.: Integrated modelling for forecasting weather and air quality: A call for fully coupled approaches, Atmos. Environ., 45, 38, 6845-6851, December 2011.

Grell, G. A. and Devenyi, D.: A generalized approach to parameterizing convection combining ensemble and data assimilation techniques, Geophys. Res. Lett., 29, 1693, doi:10.1029/2002GL015311, 2002.

Grell, G. A., Dudhia, J., and Stauffer, D. R.: A description of the fifth-generation Penn State/NCAR Mesoscale Model (MM5), National Center for Atmospheric Research Tech. Note NCAR/TN-398+STR, 138, 1994.

Grell, G. A., Peckham, S. E., Schmitz, R., McKeen, S. A., Frost, G.,
Skamarock, W. C., and Eder, B.: Fully coupled 'online' chemistry within the WRF model, Atmos. Environ., 39, 6957-6975, 2005.

Hess, M., Koepke, P., and Schult, I.: Optical Properties of Aerosols and clouds: The software package OPAC, Bull. Am. Met. Soc., 79, 831-844, 1998.

Hong, S. Y., Dudhia, J., and Chen, S. H.: A revised approach to the ice microphysical processes for the bulk parameterization of clouds and precipitation, Mon. Weather Rev., 132, 103-120, 2004.

Kain, J. S.: The Kain-Fritsch convective parameterization: an update, J. Appl. Meteorol., 43, 170-181, 2004.

Jacobson, M. Z., Lu, R., Turco, R. P., and Toon, O. B., Development and application of a new air pollution modeling system. Part I: Gas-phase simulations, Atmos. Environ., 30B, 1939-1963, 1996.

Larson, J., Jacob, R., and Ong, E.: The model coupling tookit: a new Fortran 90 toolkit for building multiphysics parallel coupled models, Int. J. High Perf. Comput Appl., 19, 277-292, 2005.

Mathur, R.: Estimating the impact of the 2004 Alaskan forest fires on episodic particulate matter pollution over the eastern United States through assimilation of satellite-derived aerosol optical depths in a regional air quality model, J. Geophys. Res., 113, D17302, doi:10.1029/2007JD009767, 2008.

Mathur, R., Xiu, A., Coats, C., Alapaty, K., Shankar, U., and Hanna, A., Development of an Air Quality Modeling System with Integrated Meteorology, Chemistry, and Emissions, Proc. Measurement of Toxic and Related Air Pollutants, AWMA, Cary, NC, September, 1998.

Michalakes, J. G.: RSL: A Parallel Runtime System Library for Regular Grid Finite Difference Models using Multiple Nests. Tech. Rep. ANL/MCS-TM-197, Mathematics and Computer Science Division, Argonne National Laboratory, Argonne, 1994.

Michalakes, J. G., Dudhia, J., Gill, D., Klemp, J., and Skamarock, W., Design of a next-generation regional weather research and forecast model. River Edge, New Jersey: Towards Teracomputing, World Scientific, 117-124, 1998.

Michalakes, J. G., Wang, W., Barker, D., Bruyere, C., Dudhia, J., and Gill, D.: User's guide for Advanced Research WRF (ARW) modeling system version 2, National Center for Atmospheric Research, http://www.mmm.ucar.edu/wrf/users, 2005.

Moeng, C. H., Dudhia, J., Klemp, J., and Sullivan, P., Examining Two-Way Grid Nesting for Large Eddy Simulation of the PBL Using the WRF Model, Mon. Weather Rev., 135, 2295-2311, June 2007.

Otte, T. L. and Pleim, J. E.: The Meteorology-Chemistry Interface Processor (MCIP) for the CMAQ modeling system: updates through MCIPv3.4.1, Geosci. Model Dev., 3, 243-256, doi:10.5194/gmd-3-243-2010, 2010.

Otte, T. L., Pouliot, G., Pleim, J. E., Young, J. O., Schere, K. L., Wong, D. C., Lee, P. C. S., Tsidulko, M., McQueen, J. T., Davidson, P., Mathur, R., Chuang, H.-Y., DiMego, G., and Seaman, N. L.: Linking the Eta Model with the Community Multiscale Air Quality (CMAQ) modeling system to build a national air quality forecasting system, Weather Forecast., 20, 367-384, 2005.

Pleim, J. E.: A combined local and nonlocal closure model for the atmospheric boundary layer. Part I: model description and testing, J. Appl. Meteorol. Clim., 46, 1383-1395, 2007.

Pleim, J. E.: A combined local and nonlocal closure model for the atmospheric boundary layer. Part II: application and evaluation 
in a mesoscale meteorological model, J. Appl. Meteorol. Clim., 46, 1396-1409, 2007.

Pleim, J. E. and Gilliam, R.: An indirect data assimilation scheme for deep soil temperature in the Pleim-Xiu land surface model, J. Appl. Meteorol. Clim., 48, 1362-1376, 2009.

Pleim, J. E. and Xiu, A.: Development of a land surface model. Part II: data assimilation, J. Appl. Meteorol., 42, 1811-1822, 2003.

Pleim, J. E. and Chang, J. S.: A non-local closure model for vertical mixing in the convective boundary layer, Atmos. Environ., 26A, 965-981, 1992.

Skamarock, W. C., Klemp, J. B., Dudhia, J., Gill, D. O., Barker, D. M., Duda, M. G., Huang, X.-Y., Wang, W., and Powers, J. G.: A description of the Advanced Research WRF version 3, National Center for Atmospheric Research Tech. Note, NCAR/TN475+STR, 113 pp., 2008.

Stauffer, D. R., Seaman, N. L., and Binkowski, F. S.: Use of fourdimensional data assimilation in a limited-area mesoscale model. Part II: effects of data assimilation within the planetary boundary layer, Mon. Weather Rev., 119, 734-754, 1991.
Wang, Q., Fu, Q., Wang, Z., Wang, T., Liu, P., Lu, T., Lin, C., Duan, Y., and Huang, Y.: Application of Ensemble Numerical Model System on the Air Quality Forecast in Shanghai, Environmental Monitoring and Forewarning, 2, 4 August 2010.

Xiu, A., Mathur, R., and Coats, C.: On the development and testing of an air quality modeling system with integrated meteorology, chemistry, and emissions, Proceedings, Symposium on Interdisciplinary Issues in Atmospheric Chemistry, Dallas, TX, Am. Meteorol. Soc., 117-120, 1999.

Xiu, A. and Pleim, J. E.: Development of a land surface model. Part I: application in a mesoscale meteorological model, J. Appl. Meteorol., 40, 192-209, 2001.

Zhang, Y.: Online-coupled meteorology and chemistry models: history, current status, and outlook, Atmos. Chem. Phys., 8, 28952932, doi:10.5194/acp-8-2895-2008, 2008. 